\title{
Chylous Ascites after Abdominal Aortic Surgery, Clinical Case Presentation, and Literature Review
}

\author{
Héctor Conrado Jimenez ${ }^{1, *}$, Edwin Romero ${ }^{2}$, Lorena Polania ${ }^{3}$, Daniel Felipe Reyes ${ }^{4}$ and Manuel Alberto Mora ${ }^{5}$ \\ ${ }^{1}$ Resident of fourth year of General Surgery, Specialist in Epidemiology, South Colombian University, Colombia \\ ${ }^{2}$ General Surgeon, Specialist in Peripheral Vascular Surgery, Del Bosque University, Colombia \\ ${ }^{3}$ General Physician, University Hospital Hernando Moncalenao Perdomo, Colombia \\ ${ }^{4}$ Department of Rural Medical Research, University Hospital Hernando Moncaleano Perdomo, Colombia \\ ${ }^{5}$ General Surgeon, Specialist in Peripheral Vascular Surgery, University Hospital Hernando Moncaleano Perdomo, Colombia
}

*Corresponding author: Héctor Conrado Jimenez, Resident of fourth year of General Surgery, Specialist in Epidemiology, South Colombian University, Colombia, E-mail: heconjisan@gmail.com

Received: 15 Jul, 2019 | Accepted: 29 Jul, 2019 | Published: 02 Aug, 2019

Citation: Jimenez HC, Romero E, Polania L, Reyes DF, Mora MA (2019) Chylous Ascites after Abdominal Aortic Surgery, Clinical Case Presentation, and Literature Review. J Surg Open Access 5(3): dx.doi.org/10.16966/2470-0991.185

Copyright: (C) 2019 Jimenez HC, et al. This is an open-access article distributed under the terms of the Creative Commons Attribution License, which permits unrestricted use, distribution, and reproduction in any medium, provided the original author and source are credited.

\section{Abstract}

Chylous ascites is a low-frequency post-operative complication, mainly associated with oncological procedures and radical lymphadenectomies. The management of chylous ascites requires knowledge of the anatomy and physiology of the cisterna chyli. A multidisciplinary approach is required to optimize outcomes. We present a 59-year-old male where chylous ascites following open abdominal aortic aneurysm repair was successfully treated.

Keywords: Chylous ascites; Abdominal aorta; Surgery; Fistula; Management

\section{Introduction}

Chylous ascites is defined as the accumulation of milky white lymph fluid in the peritoneal cavity. It was first described by Morton [1] in 1961 and since then, its incidence has increased, presenting currently 1 case for every 187,000 hospitalizations, this is due to the realization of a greater number of abdominal surgeries, and also the increase the survival in cirrhotic and oncological patients; pathologies that can cause this type of ascites [2]. The most frequent etiologies in western countries correspond to neoplasms and liver cirrhosis, although it can occur also after abdominal surgery $[2,3]$.

Patients manifest acute abdomen, bloating, chronic malnutrition and weight loss. This situation is associated with important negative consequences, a nutritional deterioration, and immunosuppression, making it necessary to combine dietary, pharmacological and surgical treatments. We present a 59-year-old man who developed chylous ascites after an open repair of the abdominal aorta, with a satisfactory response to surgical treatment.

\section{Clinical Case}

A 59-year-old male patient with a history of hypertension and heavy smoking $>20$ packs per year, was presented in the emergency service with the symptomatology of 2 years of evolution of abdominal pain in the epigastrium and mesogastrium, radiated to the genital area and testicles; with exacerbation 12 hours before admission.
It is assessed in the emergency department where it is found with a tendency to tachycardia, and normotensive upon admission. Abdominal ultrasound was performed with evidence of aneurysmal dilation of the infrarenal segment of the abdominal aorta (67.1 $\times 69.4 \times 98.3 \mathrm{~mm}$ ) that extends to the bifurcation of the common iliac arteries, without a dissection flap, for this reason, is requested an abdominal CT angiography (Figure 1). Based on the paraclinical images, the diagnosis of symptomatic Abdominal Aorta Aneurysm is established, with an indication of open surgical management by the age of the patient. It is valued by the vascular surgery service who considers it necessary to replace the abdominal aorta with a bifurcated dacron graft with anastomosis to the common iliac arteries. The patient presented an adequate clinical evolution and was discharged on day 10 of the postoperative period.

On day 20 of the postoperative period, he consulted a health center of the second level with symptoms of abdominal pain associated with fever and bloating, is order an abdominal ultrasound, finding multiple intraperitoneal collections. Due to acute abdominal pain, it is decide to perform exploratory laparotomy, during the procedure the following were presented surgical findings: milky peritoneal fluid with multiple lax septations. For suspicion of surgical-site infection, the patient there referred to a center of major complexity.

The patient is admitted at the second postoperative day of reintervention, is order a CT scan of the abdomen with double contracture (Figure 2). Due to the findings, it was decided to perform 

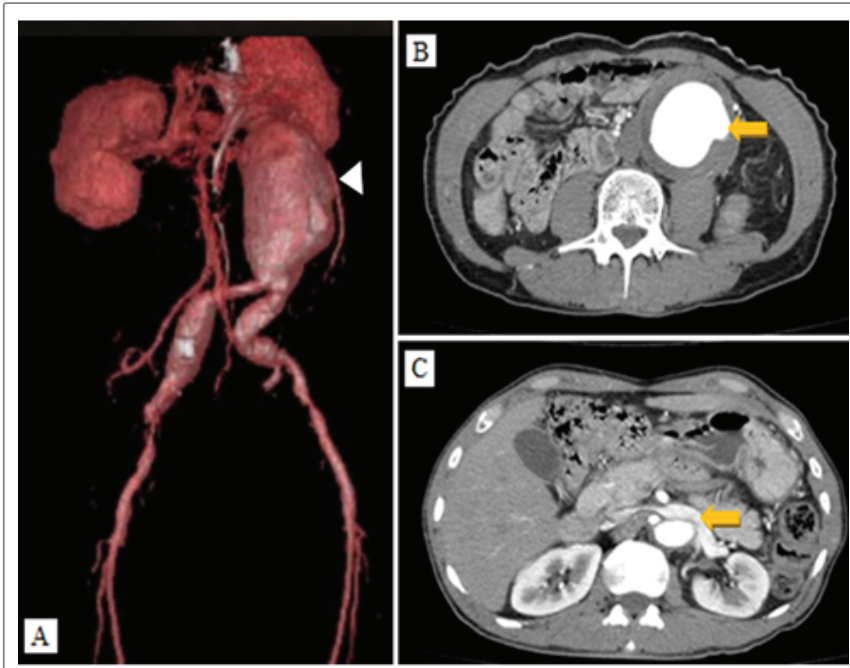

Figure 1: A: Angio CT scan of the thoracoabdominal. Fusiform dilation of the infrarenal abdominal aorta is evident before the bifurcation of the iliac arteries (white arrow) B,C: Abdominal CT The dilation of the infrarenal abdominal aorta of more than $7.8 \mathrm{~cm}$ in diameter with associated involvement of the bilateral iliac arteries (orange arrows) is evident in both cuts.

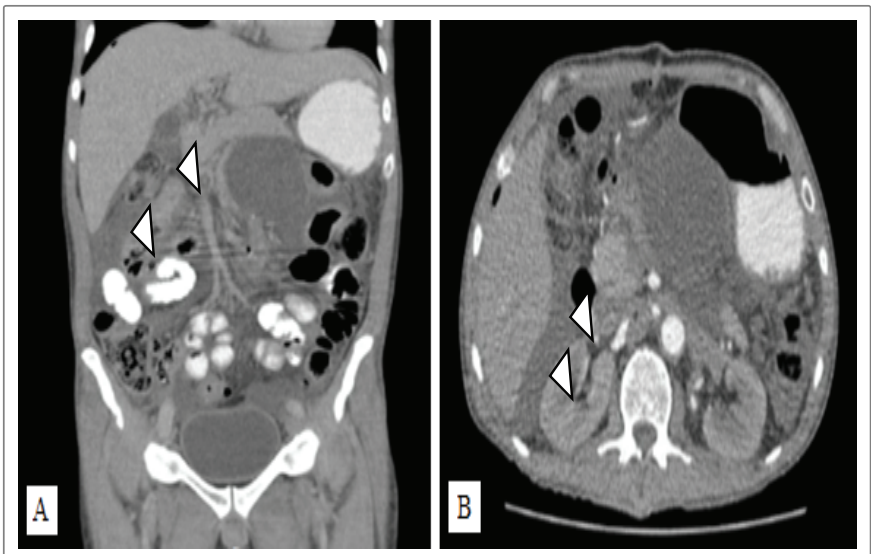

Figure 2: TAC of abdomen $(A, B)$ Intraperitoneal collections in upper hemi abdomen and right iliac fossa, which does not appear to be related to the aneurysmal sac (Arrows). Moderate ascites.

drainage of peritoneal collections guided by ultrasound, obtaining $300 \mathrm{ml}$ of milky-looking fluid that suggested chyloperitoneum, with total drainage of more than $1200 \mathrm{ml}$ in 24 hours (Peritoneal fluid study reports: triglycerides $840 \mathrm{mg} / \mathrm{dl}$ and amylase in liquid $983 \mathrm{mg} / \mathrm{dl}$ ), with these parameters the diagnosis of Chyle Fistula was considered.

A multidisciplinary management was initiated by Vascular SurgeryGeneral Surgery and Metabolic Support, following the SNAP10 (Sepsis control, Nutritional management, Anatomical characteristics of the quilose fistula, Surgical medical Plan) strategy, a broad-spectrum antibiotic, management was installed, in addition to total parenteral nutrition and infusion of somatostatin to reduce the flow in the chyle cistern. On the second day of the start of the described operation, the liquid drainage is reduced to $500 \mathrm{ml} / 24$ hours.

However, the patient subsequently persisted with increased production progressively until reaching $1300 \mathrm{ml} / 24$ hours on a ninth day; associated with this, it presents clinical deterioration, systemic inflammatory response and ventilatory compromise, for which reason it is transferred to the intensive care unit with vasopressor support; during his stay in the unit, a central venous catheter-associated bacteremia with microbiological isolation of an Enterobacter aerogenes was documented, so treatment with meropenem and polymyxin B indicated by the infectious disease service was initiated.

Due to the persistence of high losses of the peritoneal catheter (1900 $\mathrm{ml}$ in 24 hours), and not having lymphoscintigraphy for the study of chyle fistula, in addition to the persistence of sepsis despite management, it was decided to carry out joint surgical management by the vascular surgery and general surgery services; an exploratory laparotomy is performed with the following findings: retroperitoneal collection, which is drained, identifying the site of lymphatic leak, with subsequent ligation performed with 3-0 prolene-trapping points with drainage resolution, leaving Blake's drain in the management by the vascular surgery and general surgery services; an exploratory laparotomy is performed with the following findings: retroperitoneal collection, which is drained, identifying the site of lymphatic leak, with subsequent ligation performed with 3-0 prolene-trapping points with drainage resolution, leaving a Blake's drain in the zone II left retroperitoneal with left flank output. The patient presented on the second day of postoperatory, clinical improvement with control of inflammatory response, without requiring vasopressor support, peritoneal liquid of sero-bloody appearance of $800 \mathrm{ml}$ in 24 hours. It presents a good postoperatory evolution with progressive reduction of drained liquid on day 15 of its postoperatory (Figure 3), with an expenditure of $0 \mathrm{ml} / 24$ hours, in addition to the adequate tolerance to the oral route and the complete antibiotic treatment, so it is removed abdominal drain and discharge from the institution.

\section{Discussion}

Chylous ascites is a rare entity, characterized by the accumulation of chyle (intestinal lymph), at level of the peritoneal cavity, secondary to an alteration or loss of the continuity of the lymphatic vessels. There are several causes, among which are abdominal and/or retroperitoneal surgeries, malignant intraperitoneal tumors, spontaneous bacterial peritonitis, filariasis cirrhosis, among others [2].

The lymphatic system consists of a network of lymph nodes and vessels that carry lymph through certain organs, tissues, and red bone marrow. This system is developed at the end of the fifth week

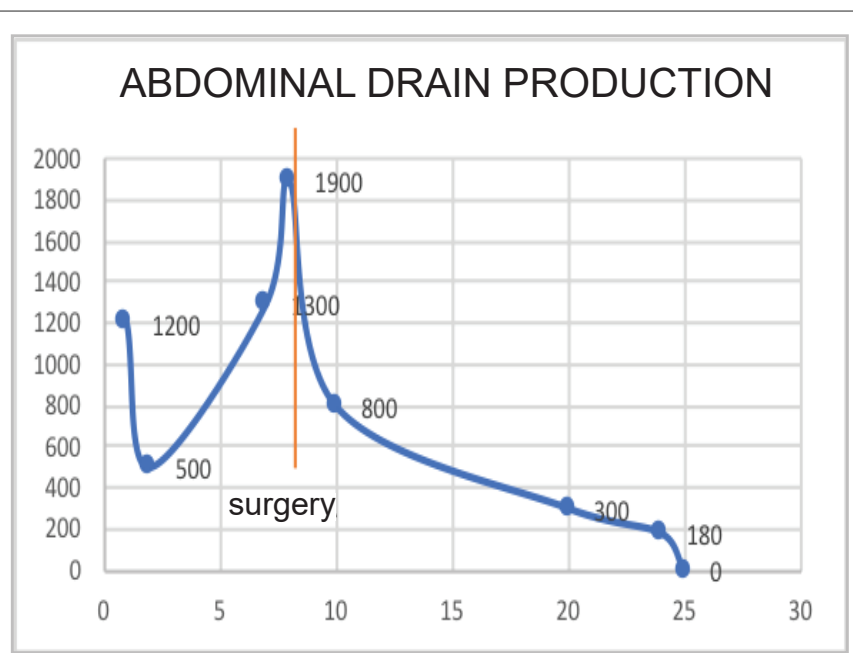

Figure 3: Abdominal drainage behavior. The orange line marks the pre and postsurgical. 
of gestation, from the sixth to the ninth week six lymphatic sacs, two jugulars, two iliac, one retroperitoneal and the Pecquet cistern at the level of the adrenal glands are formed. The Pecquet cistern communicates with the jugular sacs through large channels (the right and left lymphatic ducts) that then join together forming the definitive thoracic duct that drains the venous system at the jugular subclavian angle at the base of the neck. This system is important to fulfill the basic functions; drainage of interstitial fluid, transport of lipids, proteins, macromolecules and has an important function in the immune response [4].

The surgical procedures most frequently associated with chylous ascites are first of all repair of aortic aneurysms, followed by lymphadenectomies, cava inferior vein resection, catheter implantation for peritoneal dialysis, Nissen fundoplication, nephrectomy and liver transplantation. Kaas R, et al. [2] studied the incidence of chylous ascites in 1,103 surgical operations of the abdominal cavity and found that 12 patients (1.1\%) presented chylous ascites, all of them operated on for cancer, with lymphadenectomy being the most risky procedure [2]. Abdominal aortic surgery is a frequent procedure with clear indications; despite the anatomical relationship between the abdominal aorta and the chyle cistern, aortic surgery is an infrequent cause of chylous ascites. There are several theories that try to explain the reason of this low relation, one of them is the one of Pabst TS, et al. [5], who in his work describes only 27 cases, and suggested some risk factors for the presentation of chylous ascites in a patient with abdominal aorta surgery, including: Increase in the production of chyle and pre-existing involvement of lymphatic drainage to the abdominal cavity, a history of abdominal trauma, aneurysmal dilation, endothelial hyperplasia of the thoracic duct or obstruction of the subclavian vein. It is important to be clear about the main indications for performing open laparotomy, such as closed abdominal trauma, penetrating trauma, severe abdominal pain, unexplained persistent jaundice, chronic bleeding or ascites of unknown cause, among others, however it is highlighted that the surgical option should be chosen as long as conservative management has not worked.

The clinical presentation of chylous ascites is insidious; it may appear between 7 and 120 days postoperatory in patients with or without a history described, with abdominal pain associated with progressive abdominal distension and orthopnea [5]; chylous ascites secondary to postoperative complications can occur early (first week) due to rupture of the lymphatic vessels or late (weeks or months later) due to adhesions or extrinsic compression of the lymphatic vessels [6]. It can also be classified according to the impact of the adjacent portal system as portal or non-portal, the present case corresponds to the non-portal entity. In our patient, the clinical data were similar and presented on day 20 of postoperative, at which time he had already adequately tolerated the normal diet, which evidently led to an increase in lymphatic flow from $1 \mathrm{ml} / \mathrm{min}$ (fast) to $250 \mathrm{ml} / \mathrm{min}$ with the intake [3]. In relation to the diagnosis, it is indispensable the initial realization of a total abdominal ultrasound, with the aim of confirming intra-abdominal collections, in case of not clarity and suspicion alterations at the level of the vascular graft, it is necessary the realization of an abdominal CT with double contrast.

After this, diagnostic paracentesis guided by ultrasound should be considered to obtain a sample of peritoneal fluid and perform the respective studies to confirm the diagnosis. Generally, this fluid has milky characteristics, with an alkaline $\mathrm{pH}$, total proteins $>3 \mathrm{~g} / \mathrm{dl}$, triglycerides $>200$ and confirmatory $>1000 \mathrm{mg} / \mathrm{dl}$ and a low amount of lymphocytic predominant leukocytes [7].
In this patient, due to his consultation in the second level, abdominal ultrasound was performed early, however, due to acute abdominal symptoms; he was operated prematurely, without having data from the laboratory studies of the drained fluid. Subsequently, after the assessment in the third level by vascular surgery, the management was refocused by performing CT of the abdomen, abdominal puncture guided by ultrasound with installation of pigtail-type peritoneal catheter, and study of drained fluid, which reported high levels of amylase: $983 \mathrm{mg} / \mathrm{dl}$ and triglycerides: $840 \mathrm{mg} / \mathrm{dl}$. The lymph is rich in proteins, triglycerides, and lymphocytes ( $95 \%$ of the lymph content), so that serious lesions can cause hypoproteinemia due to the loss of albumin, fibrinogen, and immunoglobulins and, in addition, generate depletion of the reserves of fat and fat-soluble vitamins and even due to the important loss of lymphocytes, can to generate a state of lymphopenia and thus predisposition to infectious processes as in the case presented [8].

Regarding the treatment of chylous ascites, there are several proposals: repeated paracentesis, use of diuretics, re intravenous infusion of ascitic fluid, creation of peritoneum-venous shunts, diet and parenteral nutrition [9], however, currently, the mainstay of chylous ascites management, is aimed at the SNAP [10] (Sepsis control, Nutritional management, Anatomical characteristics of the quilose fistula, Surgical medical Plan) strategy, in which initial metabolic control, electrolyte stabilization, oral suspension and/or enteral nutrition low in fats and long-chain triglycerides and/or total parenteral nutrition, and use of somatostatin or its analogs and in case of no adequate response. About this medicine, the exact mechanism of how it acts is not fully understood. Somatostatin is a widely distributed polypeptide, with neuromodulator action at the central nervous system level; decreases the intestinal absorption of fats, lowers the concentration of triglycerides in the thoracic duct and decreases the flow of lymph in the main lymphatic ducts, also decreases gastric, pancreatic and intestinal secretion, inhibits the motor activity of the intestine, decreases the process of intestinal absorption and decreases splanchnic blood flow [11]. Huang Q, et al. [12] report that the use of somatostatin with should be first-line therapy for chylous ascites of different causes and should be initiated as soon as possible. Andrés AGC, et al. [11] conclude that the use of octreotide has been shown to be effective and non-invasive, reduces morbidity and mortality, hospitalization and the cost generated by complications of more aggressive treatment, without information on side effects being collected [13].

The response to conservative management revolves around $75 \%$ of cases [3]. The use of lymphogamagraphy as a study to determine the clear origin of the leak and as the last option the surgical management to perform the mechanical control of the chyle leak, however, in this case, we do not have this resource. In the case reported, due to the suspicion of a chylous fistula, management was initiated following the SNAP strategy previously mentioned. Antibiotic coverage, oral suspension, nutritional parenteral support, infusion with somatostatin and percutaneous drainage of the intra-abdominal collections with peritoneal pigtail-type catheter were started. However, due to poor response and non-availability of lymphography, surgical management was performed with clear identification of the leak site and surgical control of the same with simple raffia, leading the patient to a satisfactory evolution and discharge with strict nutritional recommendations. Diet rich in proteins, low in fat and with mediumchain triglycerides.

It is important to keep in mind that there are different surgical procedures in cases of treatment of chylous ascites, less invasive such 
as; peritoneal shunt, angiography with embolization of the basal lymphatic system and transjugular intrahepatic portosystemic shunt (which is found in a stent or stent (probe) to attach the portal veins to the blood vessels), thus reducing the pressures at the level of the portal venous bed. And with this reduce lymphatic leakage.

In relation to mortality, it can reach rates of up to $18 \%[3,6]$, and even higher, depending on the co-morbidities of the patient. In the aforementioned case, in spite of the patient's co-morbidities and the associated bacteremia, a good outcome was presented and he could be discharged.

\section{Conclusions}

The AQ is an infrequent entity, it is necessary to know its causes and have a clear knowledge of the anatomy of the abdominal lymphatic system to minimize the risk of intraoperative injuries, since they increase the morbidity and mortality of patients by up to $18 \%$, due to the need for reintervention and additional management.

\section{Conflict of Interests}

We declare that we have no conflicts of interest.

\section{References}

1. Browse NL, Wilson NM, Russo F, Al-Hassan H, Allen DR (1992) Aetiology and treatment of chylous ascites. Br J Surg 79: 1145-1150.

2. Kaas R, Rustman LD, Zoetmulder FA (2001) Chylous ascites after oncological abdominal surgery: incidence and treatment. Eur J SurgOncol 27: 187-189.

3. Aalami OO, Allen BD, Organ CH Jr (2000) Chylous ascites: a collective review. Surgery 128: 761-778.
4. Pansky B (2008) Development of the Lymphatic System. Medical embryology.

5. Pabst TS $3^{\text {rd }}$, Mclntyre KE Jr, Schilling JD, Hunter GC, Bernhard VM (1993) Management of chyloperitoneum after abdominal aortic surgery. Am J Surg 166: 194-199.

6. Carnicer JO, Checa AA, Grande MLG (2000) Ascitis quilosa tras cirugía de aorta abdominal. Med Intensiva 24: 321-323.

7. Nix JT, Albert M, Dugas JE, Wendt DL (1957) Chylothorax and chylous ascites; a study of 302 selected cases. Am J Gastroenterol 28: 40-55.

8. Leibovitch I, Mor Y, Golomb J, Ramon J (2002) The diagnosis and management of postoperative chylous ascites. J Urol 167: 449-457.

9. King TC, Smith CR (1993) Chest wall, pleura, lung, and mediastinum. In: Schwartz SI, Shires GT, Spencer FC, Daly JM, Fischer JE, et al. (eds) Principles of Surgery, Companion Handbook. $7^{\text {th }}$ Edition, McGraw Hill Professional 659-777.

10. Joyce MR, Dietz DW (2009) Management of Complex Gastrointestinal Fistula. Curr Probl Surg 46: 384-430.

11. Andrés AGC, Fernandez CM, Diez FJM, Gude VDH, Anton JAP, et al. (2004) Tratamiento conservador con octreótido del quilotorax postquirúrgico. Arch Bronconeumol 40: 473-475.

12. Huang Q, Zhi-Wei J, Li N, Jie-Shou L (2004) Chylous ascites: Treated with total parenteral nutrition and somatostatin. World J Gastroenterol 10: 2588-2591.

13. Glickman RM, Isselbacher KJ (1994) Abdominal swelling and ascites. In: Isselbacher KJ (eds) Harrison's Principles of Internal Medicine. $13^{\text {th }}$ Edition, McGraw-Hill 275-277. 